\title{
On the Design of Micro-mobility for Mobile Network*
}

\author{
Junn-Yen $\mathrm{Hu}^{1}$, Chen-Fu Chou ${ }^{2}$, Min-Shi Sha ${ }^{2}$, \\ Ing-Chau Chang ${ }^{3}$, and Chung-Yi Lai ${ }^{4}$ \\ ${ }^{1}$ Graduate Institute of Networking and Multimedia \\ ${ }^{2}$ Department of Computer Science and Information Engineering \\ National Taiwan University, Taiwan, R.O.C. \\ \{sysrq, ccf, minshi\}@cmlab.csie.ntu.edu.tw \\ ${ }^{3}$ Department of Computer Science and Information Engineering \\ National Changhua University of Education, Taiwan, R.O.C. \\ icchang@cc.ncue.edu. tw \\ ${ }^{4}$ Institute for Information Industry, Taiwan, R.O.C. \\ laici@iii.org.tw
}

\begin{abstract}
The micro-mobility issue has been discussed in host mobility in the past decade while the network mobility has become increasingly popular recently. Hence we believe that developing a micro-mobility scheme for mobile network is important and a micro-mobility scheme called Micro-NEMO is proposed in this work. The Micro-NEMO can provide local movement within an administrative domain for a moving network and be compatible with NEMO basic support protocol since it is extended from HMIPv6. Furthermore, we develop an enhanced Micro-NEMO to solve the pinball routing problem. The simulation results indicate that Micro-NEMO and its enhanced scheme can achieve a better performance than other mobility schemes in terms of number of binding update, average handoff latency, end to end delay and packet overhead.
\end{abstract}

Keywords: Mobile Network, Network Mobility, Mobility Management, Micro-mobility, Mobile Router, HMIPv6.

\section{Introduction}

The deployment of wireless networks has made mobility management research field more important. The state of the art on mobility management has been categorized into macro-mobility and micro-mobility, which could be differentiated by distinct types of handoff procedure of a mobile host. Macro-mobility means that a large scale of movement of a mobile across diverse administrative domains. C.E. Perkins purposed the Mobile IP (MIP) [1, 2] which has become the major scheme for macromobility. This scheme represents a simple and scalable global mobility solution while a mobile host is moving. However, the macro-mobility scheme is not suitable for

This work was partially supported by the National Science Council and the Ministry of Education of R.O.C. under the contract No. NSC95-2221-E-002-103-MY2 and NSC95-2622E-002-018. 
Table 1. Current state of the art for mobility management

\begin{tabular}{|l|l|l|}
\hline & Host mobility & Network mobility \\
\hline Macro-mobility & Mobile IP & NEMO Basic Support Protocol \\
\hline Micro-mobility & $\begin{array}{l}\text { Hierarchical Mobile IP, } \\
\text { Cellular IP, HAWAII, etc. }\end{array}$ & Currently none \\
\hline
\end{tabular}

movement in a small scale domain or at a high speed movement because it might incur a lot of global registration procedures and this could lead to heavy signaling overheads and significant performance degradation, e.g., the handoff latency. In order to improve the performance for mobile internet users, the micro-mobility concept was purposed. Micro-mobility means that local movement of a mobile within an administrative domain. Several Protocols such as Hierarchical Mobile IP (HMIP) [3], Cellular IP (CIP) [4, 5], HAWAII [6] have been proposed for micro-mobility. This kind of protocol has the benefit of eliminating registration between mobile host and possibly distant home agent (HA) and reducing handoff latency when the mobile node is still inside same identical local coverage area. To support mobile internet users, integration of macro- and micro-mobility can achieve objectives of low handoff latency and minimal signaling cost.

In addition, differentiated by distinct types of handoff procedure, mobility management still can be discussed from the view point of number of moving mobile hosts. If the subject of discussion is about a single mobile host moving, it is called host mobility. If a set of users are moving together via a certain transportation carriage, e.g., a bus, a train, or an aircraft, the transportation carriage can be regarded as a mobile network. This type of mobility is referred to the network mobility and the moving network is called mobile network [7]. The Internet Engineering Task Force (IETF) has proposed a basic protocol for the mobile network, named Network mobility (NEMO) basic support protocol [8], to support a large scale movement of a mobile network. In NEMO, a mobile router (MR) is able to manage the mobility of a set of mobile hosts within the same mobile network. Thus, mobile hosts inside the moving network should not perceive that MR changes point of attachment and the binding update storm can be avoided. In addition, a MR might allow other MRs to associate with itself, i.e., one mobile network could get on another mobile network. This is referred to nested NEMO, which might suffer from the pinball routing problem (so-called 'dog-leg problem').

The current state of the art for mobility management is described in Table 1. We note that many works discussed with micro-mobility issue in host mobility but did not in network mobility area. Many previous works aim to cope with the pinball routing problem of network mobility without focusing on micro-mobility issue. On the other hand, several works are not compatible with NEMO and support VMN that gets off the vehicle. We believe that micro-mobility issue in network mobility is worth discussing because the vehicle might perform many local movements such as a car move inside a city or a campus. Hence, we propose a scheme called Micro-NEMO (Micro-mobility scheme for mobile network) in this paper. 
To support micro-mobility issue for the network mobility scheme, our MicroNEMO scheme could provide that a vehicle can be local movement of micro-mobility and that visiting mobile node (VMN) still can perform micro-mobility if it gets off the vehicle. In other words, our scheme is able to efficiently integrate both network mobility and micro-mobility concepts. In addition, Micro-NEMO scheme is compatible with NEMO and host micro-mobility (for VMN get off a vehicle). Furthermore, to deal with the pinball routing problem, we provide an enhanced Micro-NEMO, which is able to perform the procedure of route optimization. Simulation results have showed that Micro-NEMO and enhanced scheme are able to improve the number of binding update, average handoff latency, end to end delay, and packet overhead in comparison with other mobility schemes.

The remaining of this paper is organized as follows. First of all, we make a brief survey of related works of micro-mobility management in network mobility. The overview of Micro-NEMO, the associated handoff mechanism, and enhanced scheme for resolving pinball routing problem are explained in section 3. Simulation environment and results for performance evaluation are presented in section 4 . Finally, section 5 concludes this paper.

\section{Related Work}

Generally, there are two types of micro-mobility issues in discussion of network mobility: (1) extra-NEMO micro-mobility [9-12] and (2) intra-NEMO micro-mobility $[13,14]$. The extra-NEMO micro-mobility means that mobile network moves around the micro-domains, which is similar to host micro-mobility. The intra-NEMO micromobility is the mobility management for the internal vehicle, i.e., a single transportation is regarded as a micro-domain. Since the focus of this work is on extraNEMO micro-mobility, we do a literature survey on recent research works on extraNEMO works in the following.

A micro-mobility scheme in [9] developed by Ohnishi, which is called Ohnishi scheme in this paper, is aimed to solve the pinball routing problem in network mobility rather than to provide a micro-mobility scheme for a moving network. Here, we first briefly discuss some famous issues of micro-mobility of host mobility - HMIPv6 [3]. In HMIPv6, mobile host moving within micro-domain performs local binding update with mobile anchor point (MAP) rather than home registration. The Ohnishi scheme is extension of HMIPv6. In Ohnishi scheme, each VMN still performs binding update by itself even when VMN gets on the vehicle. Thus, the Ohnishi scheme does not include the concept of network mobility. Therefore, even Ohnishi scheme can be backward compatible with HMIPv6 and several works about micro-mobility of network mobility issue have been proposed based on Ohnishi such as [10], we still believe that Ohnishi scheme is inappropriate for network mobility and use it as one of the compared schemes in performance evaluation section.

Besides, some works such as $[11,12]$ are about extra-NEMO micro-mobility. In [11], authors proposed a route optimization methodology that uses unidirectional 
tunneling and a tree-based intra-domain routing mechanism and declare that it can be easily extended to support micro-mobility. However, it could not be backward compatible with conventional host micro-mobility. So that mobile host may not perform micro-mobility when it gets off the vehicle. Another work in [12] proposed the HMIP-B (Hierarchical Mobile IPv6 extension with buffering function) scheme, in which MAP store packets destined to the mobile hosts during the process of handoff. That is, the focus of HMIP-B is to reduce packet loss rather than to provide a micromobility scheme.

\section{Micro-mobility Scheme for Mobile Network}

\subsection{Protocol Overview}

Micro-NEMO, as depicted in Figure 1, is aimed to support simultaneous (multiple consequent) local movements within an administrative domain (micro-domain) for a mobile network. Hence, the major idea of Micro-NEMO scheme is to include the concept of micro-mobility into NEMO protocol while preserving both the characteristics of micro-mobility and NEMO protocols. That is, Micro-NEMO is designed to achieve low handoff latency, has minimal signaling cost and be transparent to all the mobile hosts within the same mobile network, i.e., mobile hosts inside that moving network will not perceive that the MR has changed point of attachment. At last, to be compatible with the NEMO basic support protocol that is extended by MIPv6 as HMIPv6, Micro-NEMO is built from HMIPv6 as well.

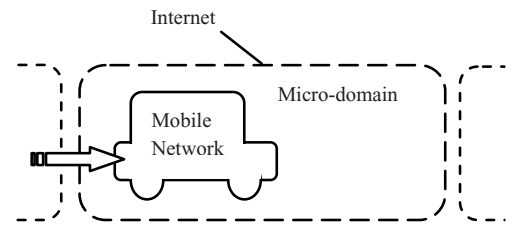

Fig. 1. Micro-mobility Scheme for mobile network

\subsection{Local Movement Scenarios for Mobile Network}

To design the Micro-NEMO, we begin with understanding the relationship between VMN and transportations. In general, there are four movement scenarios for micromobility of mobile network as shown in Figure 2. First, the location management happens when mobile network initially enters a new micro-domain as illustrated in Figure 2(a). This is similar to that a mobile host first gets into a new micro-domain. Second, a visit mobile node (VMN), which is like that a human has a mobile device such as mobile phone, gets on the transportation as illustrated in Figure 2(b). Next, Figure 2(c) depicts that the mobile network roams within a micro-domain. Finally, the case in Figure 2(d) is that VMN takes off the transportation. 


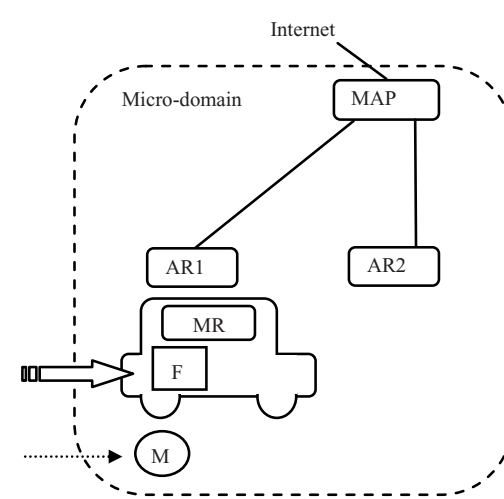

(a) Scenario 1

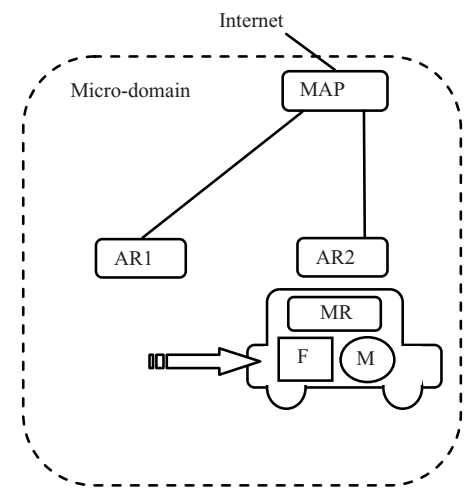

(c) Scenario 3

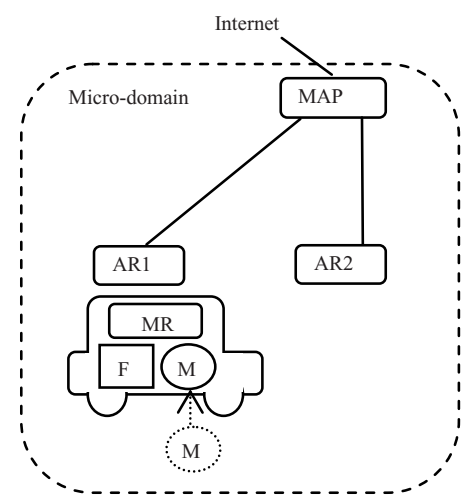

(b) Scenario 2

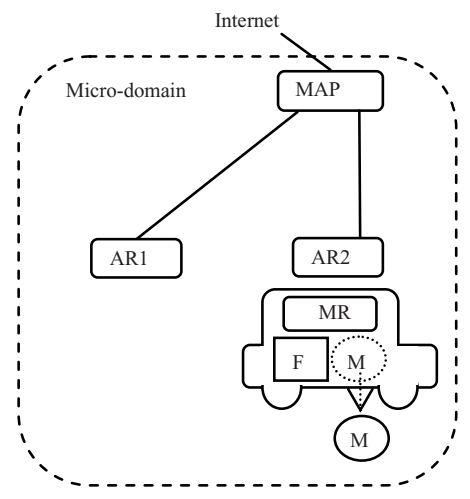

(d) Scenario 4

Fig. 2. Scenarios for Micro-NEMO

\subsection{Protocol Description}

As discussed before, there are four types of scenarios for mobile network in the micro-domain environments. In this section, we describe all the details of operations for each scenario in the following.

Scenario 1. When a vehicle (mobile network) enters a new micro-domain, the MR of that vehicle starts to perform the operation of micro-mobility scheme to allow the home agent of MR can be aware of the location of the vehicle. Figure 3 (a) and (b) shows that the signaling flow and the data delivery respectively for a vehicle entering the micro-domain at the first time. The MR of the vehicle will obtain the on-link careof-address (LCoA) and regional care-of-address (RoA), and it registers with MAP to establish a binding. Then, MR performs home registration with HA of MR. After these initial signalings are finished, that home network (home link) of the vehicle knows on which micro-domain the vehicle is. Once a $\mathrm{CN}$ wants to transmit data packet to LFN (local fixed node), the data packets pass through HA of MR, MAP and reach LMN finally. 


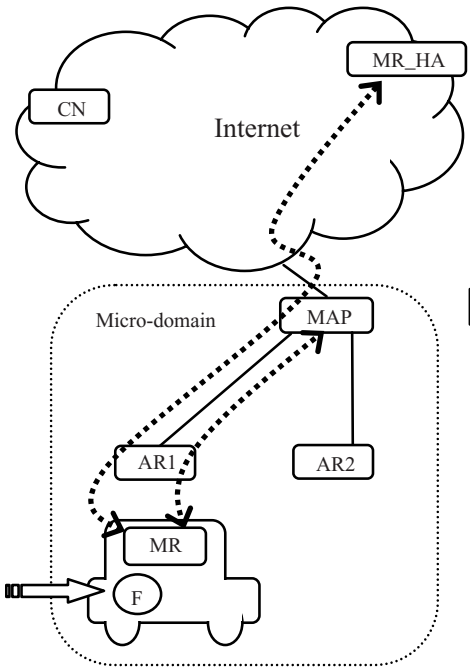

(a) Signaling flow

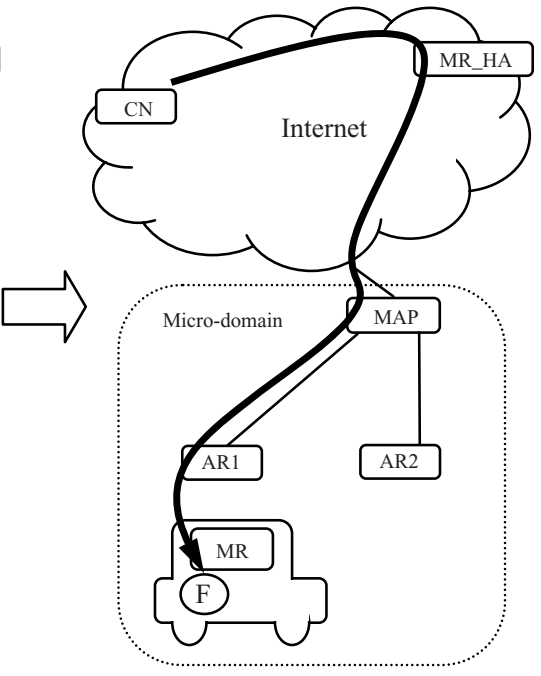

(b) Data delivery

Fig. 3. When a vehicle (mobile network) first enter a micro-domain

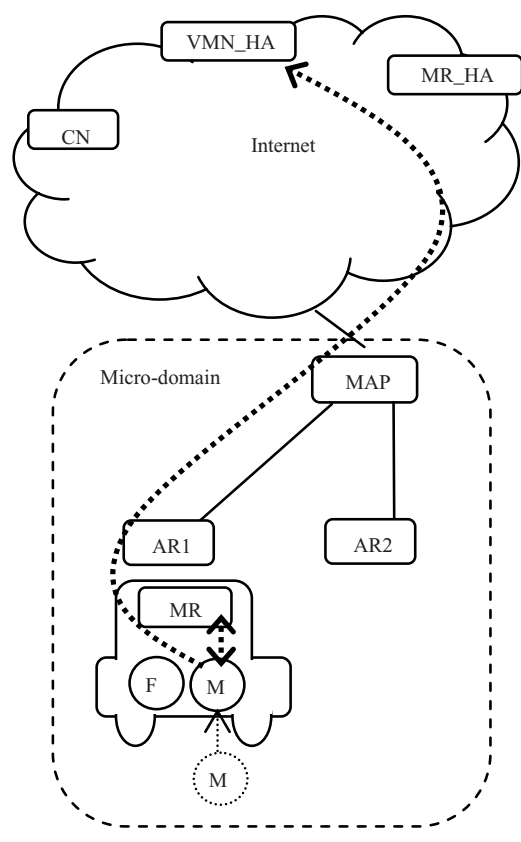

(a) Signaling flow

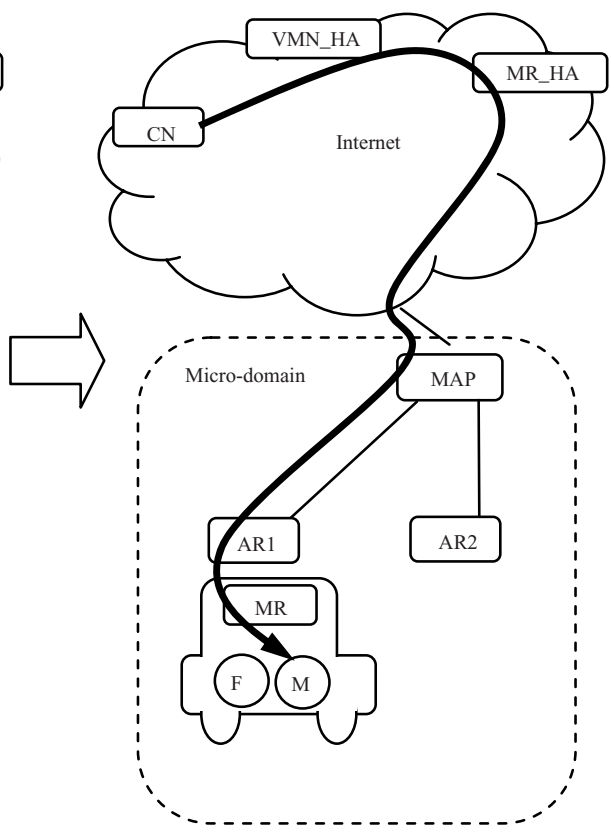

(b) Data delivery

Fig. 4. When a VMN get on the vehicle 
Scenario 2. Figure 4 shows that a VMN gets on a vehicle within a micro-domain. In this case that VMN will inform its home agent (HA) once and the mobile router (MR) of the mobile network will perform location management function on behalf of VMN(s) as the vehicle movement. After VMN obtains a CoA from MR, it starts to perform home registration once as illustrated in Figure 4(a). Afterward there is no need home registration between VMN and its HA. As for the data delivery, data packets sent by $\mathrm{CN}$ will be tunneled by the HA of VMN and the HA of MR respectively, then pass through $\mathrm{MAP}$ and arrive at VMN finally as illustrated in Figure 4(b).

Scenario 3. Based on the concept of the micro-mobility, there is no need for MR of a vehicle to perform home registration when it moves around within the same microdomain. In other words, it only needs to obtain a new LCoA and perform local binding update to the MAP in order to establish a binding between the LCoA and RCoA. In addition, all VMNs within that vehicle do not carry out any binding updates except the home registration at the first time.

Scenario 4. Lastly, when a VMN gets off the vehicle, this case is similar to that a VMN enters a new micro-domain. Since the VMN is not in a mobile network any more and should perform micro-mobility scheme by itself, VMN starts to do the process of host mobility.

\subsection{Advantage and Drawback}

In order to provide an efficient scheme integrating both micro-mobility and network mobility, we proposed a Micro-NEMO protocol. In this basic solution, we directly apply micro-mobility concept into the Micro-NEMO protocol, i.e., a vehicle only needs to perform local binding update with MAP when it moves within an administrative micro-domain. Specifically, the mobile network does not perform home registration with $\mathrm{HA}$ unless it traverses to a new micro-domain. As a consequence, the number of global binding updates could be reduced. On the other hand, Micro-NEMO protocol is corresponding to the concept of network mobility as well, i.e. the mobility of the vehicle is transparent to its residing nodes (e.g. local fixed nodes and visiting mobile nodes). However, we note that Micro-NEMO still suffers from the same drawback, i.e., the pinball routing problem in Figure 4(b), as the basic NEMO protocol does. That is, data packets will be tunneled through multiple HAs (both HAs of VMNs and HA of MR) before it arrives to the destination host. This not only results in sub-optimal end-to-end path, but also incurs heavy packet overheads. Moreover, the problem becomes more critical if the number of levels of nested NEMO increases. Next, we will purpose an enhanced Micro-NEMO protocol to deal with the pinball routing problem.

\subsection{Enhanced Approach}

We note that the pinball routing problem can be solved if the HA of the root MR knows all the binding information of child MRs and VMNs. Hence, the HA of the root $\mathrm{MR}$ is able to perform binding update with the sender $(\mathrm{CN})$ by $\mathrm{RCoA}$ of root MR. Afterward, CN could directly forward packets to VMN of the vehicle inside a micro-domain. 


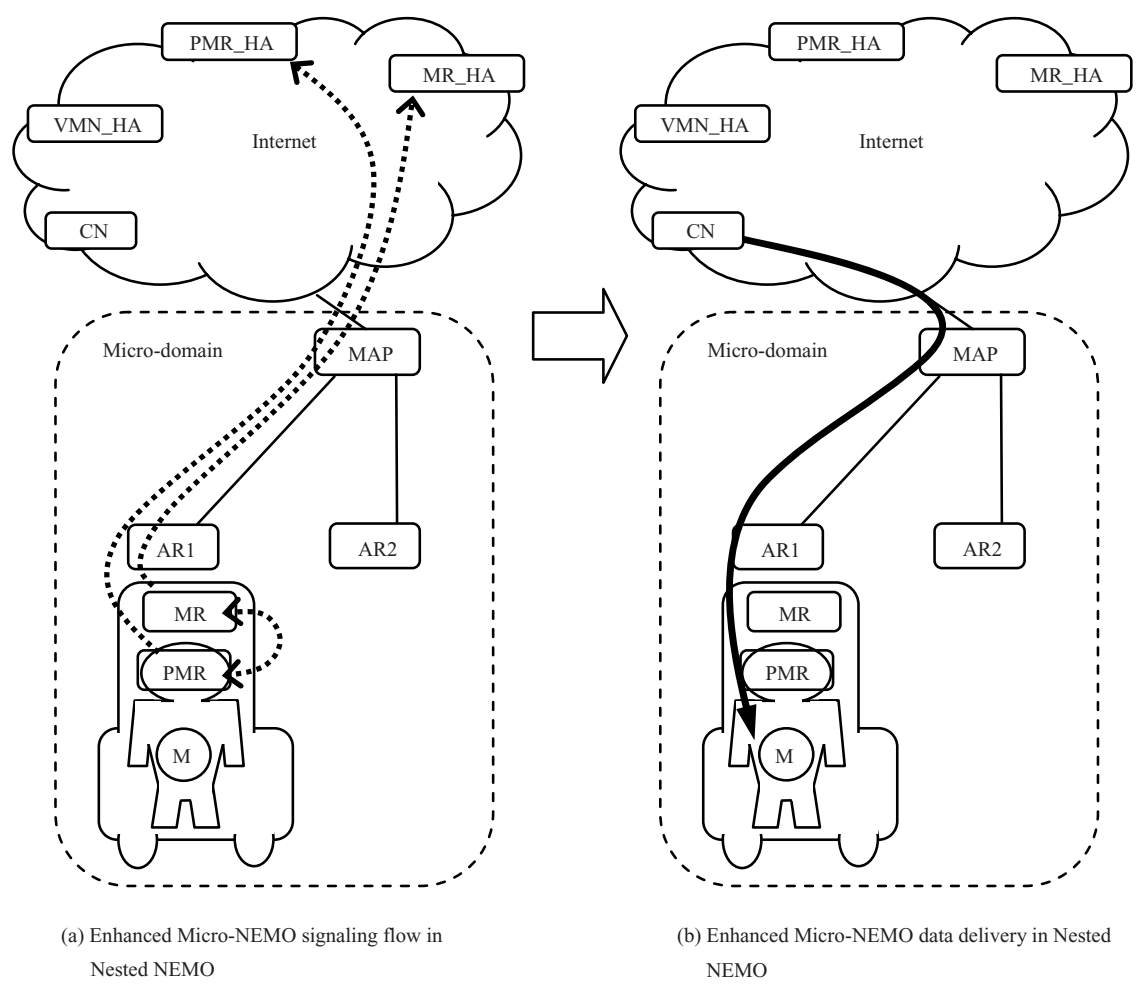

Fig. 5. Enhanced Micro-NEMO Protocol

Figure 5 shows that signaling flow and data delivery of enhanced Micro-NEMO. When a human with a personal area network (PAN) gets on a vehicle, MR needs to inform HA of MR of location information of nodes inside entering moving network and PMR (MR of PAN) also has to perform home registration with its HA as illustrated in Figure 5(a). Once HA of MR receives packet sent from HA of child node, it could perform binding update with $\mathrm{CN}$. Afterward $\mathrm{CN}$ is able to directly forward data packets through MAP to the VMN as depicted in Figure 5(b). Hence, our enhanced approach could efficiently cope with the pinball routing problem. That is, the enhanced approach could not only shorten end to end delay between sender $\mathrm{CN}$ and VMN for nested NEMO but also reduce packet overheads through getting rid of multiple tunneling.

\section{Performance Evaluation}

\subsection{Simulation Environment and Performance Criteria}

Simulation study has been conducted to evaluate the performance of Micro-NEMO as well as the enhanced approach. There are total 64 micro-domains in the simulation, 
i.e., an $8 \times 8$ mesh grids. Each micro-domain mesh grid is equipped with a MAP. Moreover, each MAP contains 16 ARs, in $4 \times 4$ sub-grids and each sub-grid has an AR. There are total 500 moving vehicles randomly scattered over all micro-domains. Each vehicle has a MR and 5 VMNs and the total number of VMN is 2500. In order to model the mobility of the vehicle, time is slotted and MoveProb(Movement Probability) is used in the simulation. MoveProb represents the probability of a vehicle leaves its current $A R$ in the next time slot. When a vehicle decides to leave the current $\mathrm{AR}$ in the next time slot, its next $\mathrm{AR}$ is randomly selected from the neighboring ARs. Details of the simulation parameter are described in Table 2.

Table 2. Simulation parameters

\begin{tabular}{|l|}
\hline \multicolumn{1}{|l|}{ MR(Vehicle)\# = 500 } \\
VMN\# = 2500, 5 VMNs per MR \\
MoveProb (Movement Probability) $=0.8$ \\
Simulation Time = 1000 time units \\
\hline \multirow{2}{*}{$\begin{array}{l}\text { Internet latency }=50 \text { time units } \\
\text { Local domain latency }=10 \text { time units } \\
\text { Backbone latency }=1 \text { time units }\end{array}$} \\
\hline
\end{tabular}

Four performance metrics are used to compare the proposed Micro-NEMO basic and enhanced scheme with other schemes and they are: (1) total number of binding updates, (2) the average handoff latency, (3) end to end delay, and (4) packet overhead. The average handoff latency is defined as the time to complete binding update after a handoff, the end to end delay is defined as the time interval for a data packet from sender to receiver, and the packet overhead is defined as the ratio of encapsulate packet headers size to total packet size.

\subsection{Simulation Results}

Figure 6 shows the total number of binding update under different schemes. To compare with basic NEMO protocol, the number of global binding update of MicroNEMO is lower than basic NEMO protocol since it could effectively integrate the idea of the micro-mobility. In addition, the total number of binding updates in both HMIP and Ohnishi scheme is higher than Micro-NEMO because they do not consider the concept of network mobility, i.e., they ignore the characteristic of the mobile network, all the mobiles hosts within the same mobile network can update their location information through a "single" binding update of MR of that mobile network. In other words, when the MR changes its access point, all mobile hosts inside the moving network will not observe that change such that the binding update storm for all MHs can be avoided.

Comparison of the average handoff latency is depicted in Figure 7. Since the proposed scheme can provide the functionality of micro-mobility, it is not surprising that the latency is lower than NEMO, HMIP and Ohnishi. Next, Figure 8 shows that the results of the end to end delay for different schemes. Since the proposed enhanced Micro-NEMO scheme is equipped with the process of route optimization, the end to 
end delay has been significantly deceased. At last, due to the same reason, i.e., the route optimization functionality, Figure 9 also shows that enhanced Micro-NEMO is much better than other schemes in terms of packet overhead.

Please note that we have performed a wide range of simulations for different parameter settings, e.g., MoveProb values, mobility pattern and vehicle speed etc. Due to the limit space of the paper, we only use the case of MoveProb $=0.8$ in the paper. Qualitatively, the similar trend still persists.

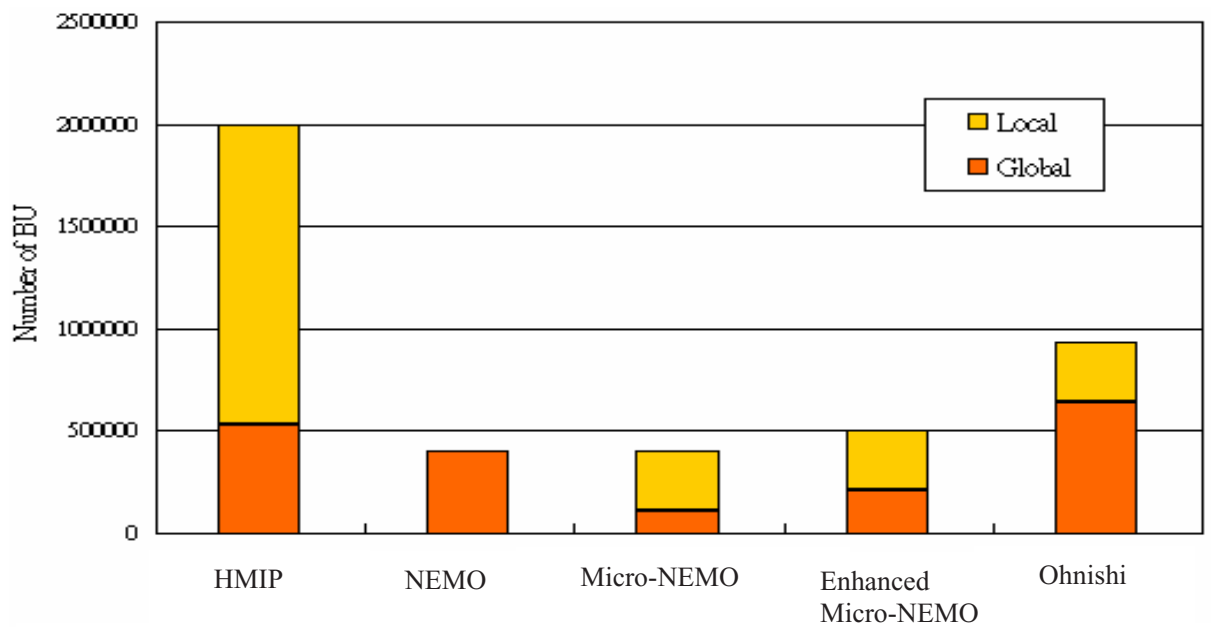

Fig. 6. Total number of binding update

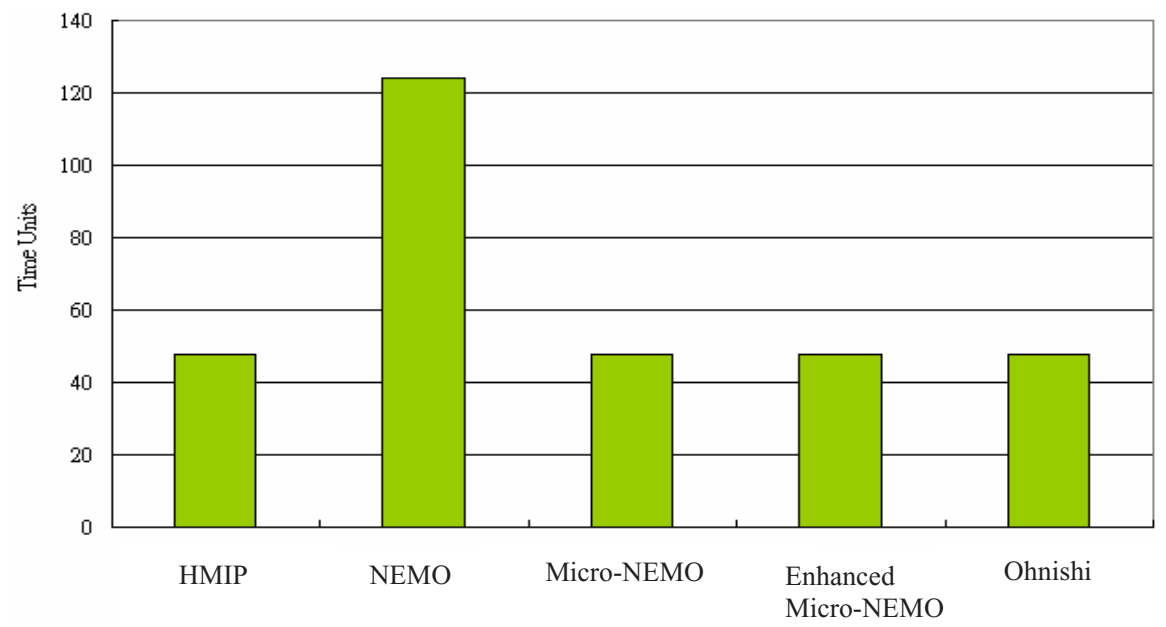

Fig. 7. Average handoff latency 


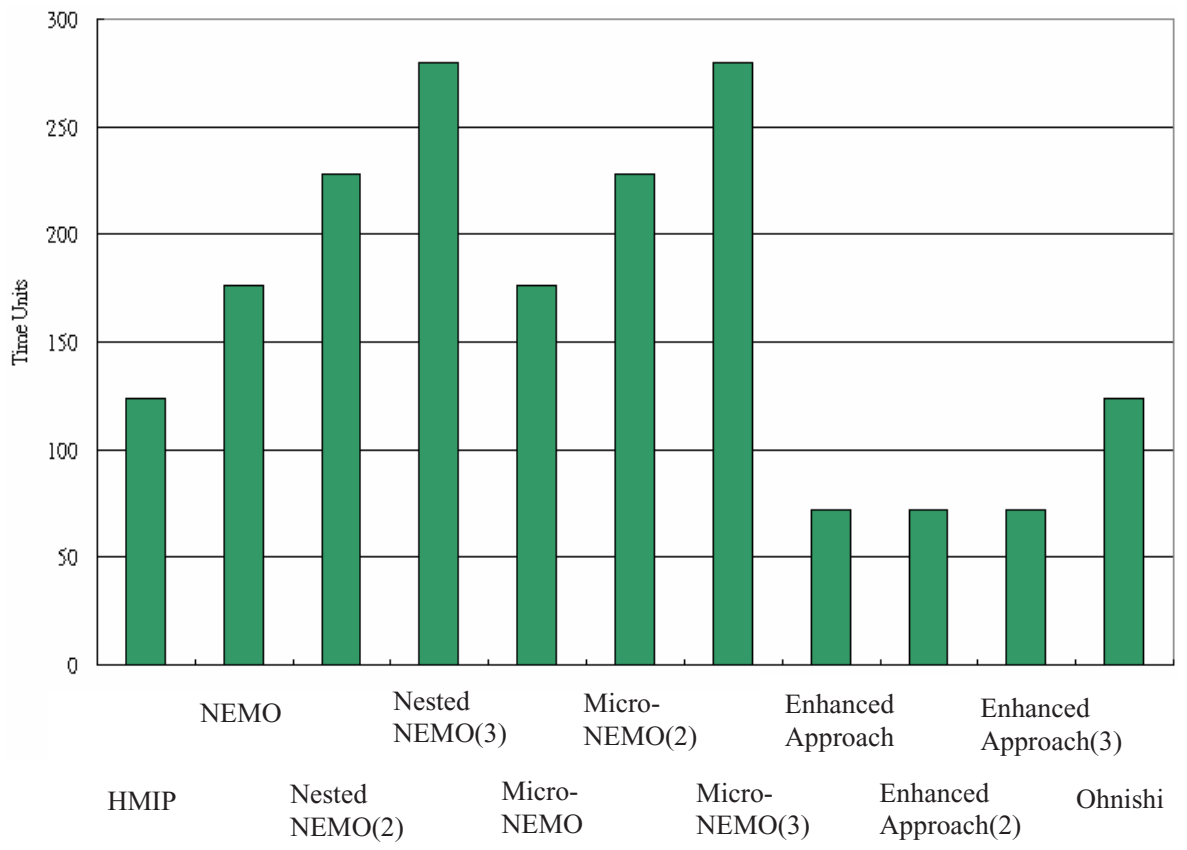

Fig. 8. End to end delay

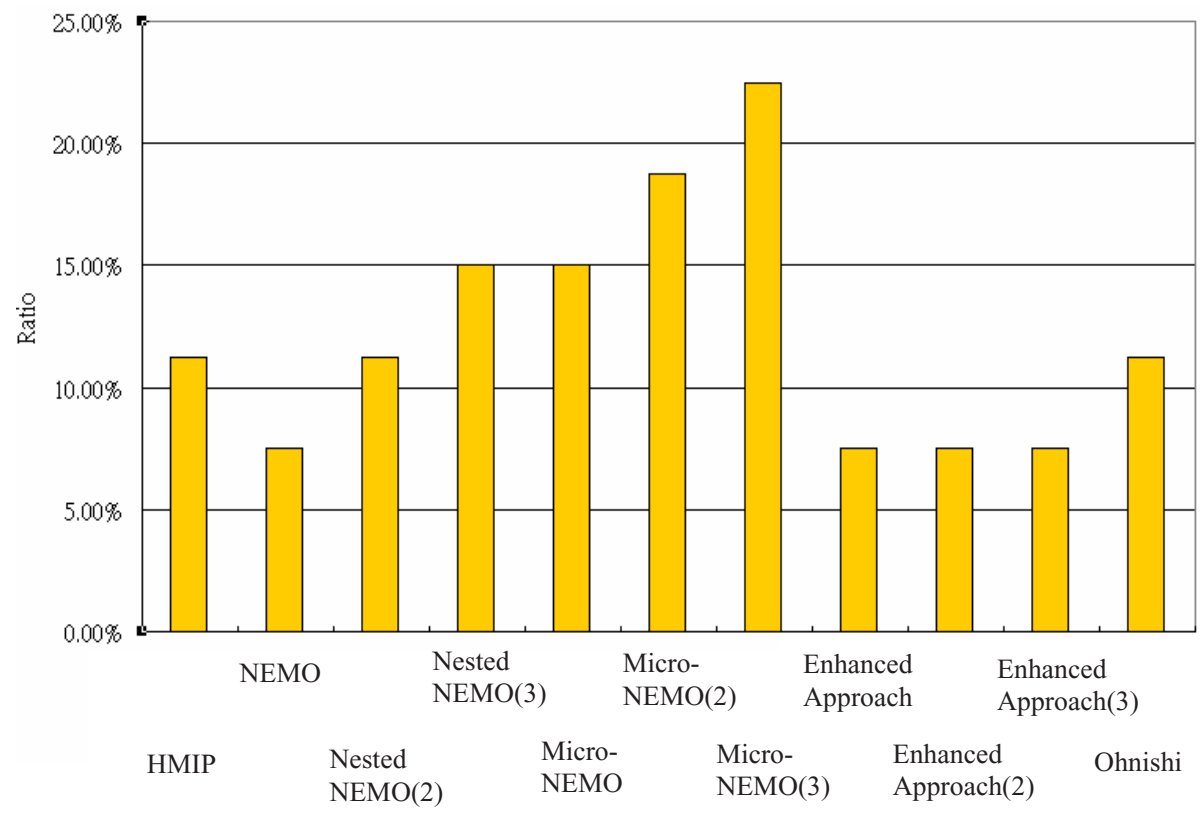

Fig. 9. Packet Overhead 


\section{Conclusion}

In the past decade, there are plenty of research works focusing on micro-mobility issue for the host mobility protocol. Since the network mobility has attracted much attention recently, we believe there is a need to support the functionality of micromobility for NEMO. In this paper, we propose a micro-mobility scheme for mobile network (Micro-NEMO). To be backward compatible with NEMO, the proposed scheme is extended from HMIPv6. The scheme can provide local movement of vehicle and integrate to micro-mobility of the single mobile host. Furthermore, to deal with the pinball routing problem, we provide an enhanced Micro-NEMO, which is able to perform the procedure of route optimization. Simulation results have showed that Micro-NEMO and enhanced scheme are able to improve the number of binding update, average handoff latency, end to end delay, and packet overhead in comparison with other mobility schemes.

\section{References}

1. Johnson, D., Perkins, C., Arkko, J.: IP Mobility Support. IETF, RFC 2002 (1996)

2. Perkins, C.: Mobile IP. IEEE Communication Magazine 35(5), 84-99 (1997)

3. Soliman, H., Castelluccia, C., El Malki, K., Bellier, L.: Hierarchical Mobile IPv6 Mobility Management (HMIPv6). IETF, RFC 4140 (2005)

4. Campbell, A., Gomez, J., Kim, S., Valko, A., Wan, C., Turanyi, Z.: Design implementation and evaluation of cellular IP. IEEE Personal Communications 7(4), 42-49 (2000)

5. Campbell, A., Gomez, J., Kim, S.: An Overview of Cellular IP. In: WCNC. Proceeding of IEEE Wireless Communications and Networking Conference, vol. 2, pp. 606-610 (1999)

6. Ramjee, R., Varadhan, K., Salgarelli, L., Thuel, S., Wang, S., Porta, T.L.: HAWAII: A domain-based approach for supporting mobility in wide-area wireless networks. IEEE/ACM Transactions on Networking 10(3), 396-410 (2002)

7. Lach, H.-Y., Janneteau, C., Petrescu, A.: Network mobility in beyond-3G systems. IEEE Communication Magazine 41(7), 52-57 (2003)

8. Devarapalli, V., Wakikawa, R., Prtrescu, A., Thubert, P.: Network Mobility (NEMO) Basic Support Protocol. IETF, RFC 3963 (2005)

9. Ohnishi, H., Sakitani, K., Takagi, Y.: HMIP based route optimization method in a mobile network. IETF, Internet Draft (2003)

10. Novak, R.: Proxy MAP for Intra-domain Route Optimization in Hierarchical Mobile IP. IEICE Transactions on Communications E89-B(2) (2006)

11. Jeong, M.-S., Park, J.-T.: Hierarchical Mobile Network Routing: Route Optimization and Micro-Mobility Support for NEMO. In: Yang, L.T., Guo, M., Gao, G.R., Jha, N.K. (eds.) EUC 2004. LNCS, vol. 3207, pp. 571-580. Springer, Heidelberg (2004)

12. Omae, K., Inouem, M., Okajima, I., Umeda, N.: Handoff performance of mobile host and mobile router employing HMIP extension. In: WCNC. Proceeding of IEEE Wireless Communications and Networking Conference, vol. 2, pp. 1218-1222 (2003)

13. Rónai, M.A., Fodor, K., Tönjes, R.: IPv6 Moving Network Testbed with Micro-Mobility Support. IST Mobile and Wireless Communications, 596-600 (summit, 2004)

14. Watanabe, Y.: A Micro Mobility Protocol for Network Mobility with Fast Handovers. In: The International Conference on Internet Networking (2006) 